OP0076

HOW TO SELF-EXAMINE FOR TENDER AND SWOLLEN JOINTS: CO-DEVELOPMENT OF A TRAINING VIDEO FOR PEOPLE WITH RHEUMATOID ARTHRITIS

C. Sharp ${ }^{1,2}$, K. Staniland ${ }^{3}$, T. Cornell ${ }^{4}$, W. Dixon ${ }^{3,5} .{ }^{1}$ The University of Manchester, Centre for Epidemiology Versus Arthritis, \& Centre for Primary Care and Health Services Research, Manchester, United Kingdom; ${ }^{2}$ Manchester Royal Infirmary, Kellgren Centre for Rheumatology, Manchester, United Kingdom; ${ }^{3}$ The University of Manchester, Centre for Epidemiology Versus Arthritis, Manchester, United Kingdom; ${ }^{4}$ Poole Hospital, Rheumatology, Poole, United Kingdom; ${ }^{5}$ Salford Royal NHS Foundation Trust (Formerly Hope Hospital), Rheumatology, Salford, United Kingdom

Background: COVID-19 has catalysed the transformation of healthcare services, with outpatient services increasingly dependent upon remote models of care. Healthcare professionals now have to make clinical assessments based on remote patient examinations. The number of tender and swollen joints a patient has drives decision-making in RA, making it particularly important that people with RA and HCPs have a shared understanding of these examinations.

Even before remote consultations became widespread, long gaps between clinic visits create challenges in enabling HCPs to form an accurate picture of disease activity over time. The REMORA (REmote MOnitoring of Rheumatoid Arthritis) app aims to address this issue by asking people with RA to track disease activity, including counting the number of tender or swollen joints, weekly(1). Data are integrated into the electronic patient record for clinicians to access with patients during clinical consultations. As part of the supporting materials for the REMORA app, we planned to develop a video to train people with RA how to examine their own joints. This video may now help meet the need created by the recent expansion in remote consultations.

Objectives: To describe the co-production, implementation and evaluation of a video to train patients how to examine their own joints.

Methods: The need for the video to fill a current gap in patient education was identified by the REMORA patient and public involvement and engagement (PPIE) group. A core working group comprising the PPIE lead, a nurse consultant, rheumatology clinicians, project and communications managers was formed. A storyboard was drafted and feedback gained from the PPIE group and wider REMORA team. Images were sourced from licenced suppliers, or co-developed with the PPIE group where necessary. No ethical approval was required as the PPIE group lead was acting as an equal member of the research term. Written informed consent was gained from video participants. Filming took place between two national lockdowns during the COVID-19 pandemic, providing a challenge to ensure social distancing and requiring the use of masks.

Results: A 15 minute video to train people with RA to self-examine for tender and swollen joints was developed. An introduction outlining the rationale behind self-examination is followed by a nurse consultant coaching an RA patient in individual joint self-examination. Shoulders, elbows, wrists, metacarpophalangeal joints, proximal interphalangeal joints and knees are included, all of which are counted in disease activity scores.

Early feedback from stakeholders has been overwhelmingly positive. The video will be publicly available on YouTube from February 2021. A survey of patients and HCPs aims to obtain more formal feedback on the video, with a view to a further iteration, if required. Leading national organisations in rheumatology will promote the video, as it supports national programmes including the British Society for Rheumatology national early inflammatory arthritis audit and ePROMS (electronic patient report outcome measure) platform, both of which include entry of patient reported tender and swollen joint counts.

Conclusion: This video was co-designed by people with RA, aiming to support self-examination of tender and swollen joint counts. Hits on YouTube and survey responses will help assess its impact. Evaluation to assess whether the video affects patients' ability to self-examine for tender and swollen joints before and after watching is planned. We hope the video will support remote consultations and help people with arthritis to better understand and self-manage their arthritis, and to have shared decision making conversations with their clinicians. REFERENCES:

[1] Austin L, Sharp CA, van der Veer SN, Machin M, Humphreys J, Mellor P, et al. Providing 'the bigger picture': benefits and feasibility of integrating remote monitoring from smartphones into the electronic health record: findings from the Remote Monitoring of Rheumatoid Arthritis (REMORA) study. Rheumatology. 2020;59(2):367-78

Disclosure of Interests: Charlotte Sharp: None declared, Karen Staniland: None declared, Trish Cornell Shareholder of: shares in Abbvie, Employee of: Abbvie, working as a Rheumatology Nurse Consultant, Will Dixon Consultant of: Google and Abbvie, unrelated to this work.

DOI: 10.1136/annrheumdis-2021-eular.1990

OP0077

THE EMERGING EULAR NETWORK (EMEUNET): AN INTERNATIONAL SURVEY REFLECTING ON A TEN-YEAR JOURNEY

M. de Hooge ${ }^{1}$, M. Hanlon ${ }^{2}$, A. Alunno ${ }^{3}$, A. Sepriano ${ }^{4}$, K. Lauper ${ }^{5}$, F. Rivellese 6 . ${ }^{1}$ Ghent University Hospital, Rheumatology, Ghent, Belgium; ${ }^{2}$ Trinity University
College, Rheumatology, Dublin, Ireland; ${ }^{3}$ University of Perugia, Rheumatology, Perugia, Italy; ${ }^{4}$ Leiden University Medical Center, Rheumatology, Leiden, Netherlands; ${ }^{5}$ University Hospitals of Geneva, Rheumatology, Geneva, Switzerland; ${ }^{6}$ Queen Mary University, Rheumatology, London, United Kingdom

Background: The Emerging EULAR NETwork (EMEUNET), founded in 2009, aims to promote education, foster research collaborations and facilitate the integration of young researchers and rheumatologists within EULAR.

Objectives: After 10 years, we aimed to interview our members to assess how EMEUNET is fulfiling its goals, understand if there are unmet needs and explore new ideas. Methods: In July 2019 a survey was circulated for 3 months among EMEUNET members via its social media channels. The survey contained 18 closed and 2 open items, in addition to general questions on age, job and country of origin and work. Results: Out of 124 total respondents, most completed all items (120/124) and only a minority had some missing items (4/124). A little over half of the respondents were female $(n=69,54.8 \%)$ and median age was 33y (range 25y-42y). Most respondents were born $(n=109,88.6 \%)$ and/or worked in Europe $(n=114,92.7 \%)$ (fig. 1) although there was also a representation from extra-European countries, which is in line with the general EMEUNET membership (https://emeunet.eular.org/map.cfm).

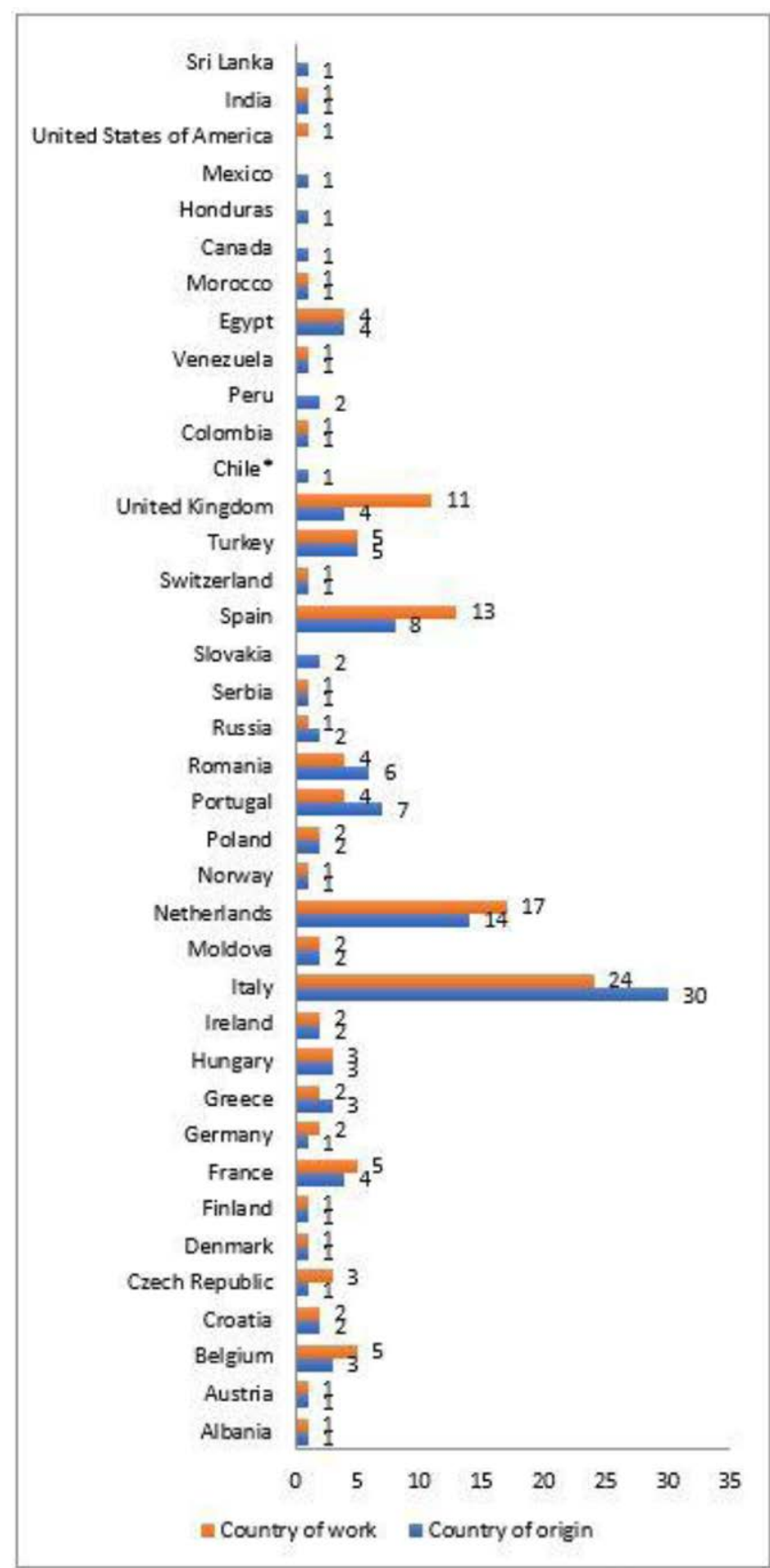

Figure 1. Country of origin and where respondents work at the time of completing the EMEUNET VISE questionnaire. 
$11(9.1 \%)$ had been EMEUNET members for less than 2 years, 81 (66.9\%) for 2-5years and 29 (24.0\%) for more than 5 years.

Figure 2 shows a clear link between the respondents' perception of EMEUNET aim(s) and the actual aims of EMEUNET set out in our mission statement (https://emeunet.eular.org/mission_statement.cfm).

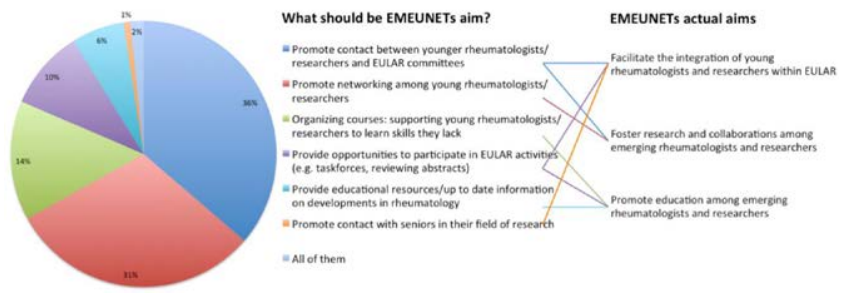

Figure 2. The links between what participants would like to see as EMEUNET aims and the aims of EMEUNET as they are now

Most of the respondents got to know about EMEUNET through a friend/colleague in Rheumatology ( $n=67,54.0 \%$ ), at EULAR/ACR annual conferences $(n=32,25.8 \%)$, via social media $(n=9,7.3 \%)$, other international meeting $(n=6$, $4.8 \%)$, national Rheumatology meeting $(n=6,4.8 \%)$ or via the EMEUNETs country liaisons $(n=4,3.2 \%)$.

29 respondents (24\%) were part of the EMEUNET working group (WG), 43\% $(n=53)$ applied before to be part of the WG, 24\% $(n=29)$ never applied and $15 \%$ $(n=19)$ did not know what the WG was. Most of the respondents who never applied to the WG thought it to be too time consuming. Only $21(16.9 \%)$ felt there was a subgroup in the WG missing and most of those $(n=8,38.1 \%)$ found this should fully focus on research collaborations, which is actually part of the general aims of EMEUNET and a focus of all Working Groups.

Only a relatively small portion of EMEUNET of respondents $(<5 \%)$ stated to have approached EMEUNET with their ideas. Although $90.2 \%(n=110)$ felt that the opportunity to submit new ideas is a good initiative, only $52.5 \%(n=.$.$) knew$ that such possibilities exists. This suggests that EMEUNET could do more to make members aware of this possibility. Additionally, while the main reasons for not contacting EMEUNET with an idea were 'not having one' $(41.9 \%)$ or having 'no time' (34.9\%), 20.9\% feels their ideas would not be received well, which is another aspect offering room for improvement.

Twice a year, EMEUNET organises networking events (NE) for their members to discuss their work in an informal setting. We found that $56 / 120(46.7 \%)$ of the respondents prefer a low-budget $(<€ 20)$ event, including an activity and the possibility to network, accompanied by drinks and bites. This is much in line with EMEUNETs past NE. In addition, past NEs were often mentioned as an example of ideal events.

Conclusion: Awareness on EMEUNET often comes from colleagues and international conferences in rheumatology, which coincides with one of the focuses of EMEUNET to increase visibility in the last 10 years. Expectations about NE are in line with the previously organised NE.

Areas for improvement are dissemination of information on the EMEUNET WG, the possibility to submit ideas -which is open to all members- and the increased focus on research collaboration, an aspect on which we are actively working as EMEUNET is fully engaged with the newly launched EULAR Virtual Research Centre.

Disclosure of Interests: None declared

DOI: 10.1136/annrheumdis-2021-eular.2706

\section{OP0078 $\quad$ HIGH MEDIAN NERVE LESION SECONDARY TO SEVERE GIANT CELL ARTERITIS (GCA)}

K. Iqbal ${ }^{1}, 2$, J. Miller ${ }^{3}$, M. Lai ${ }^{4}$, M. Charnley ${ }^{2}$, M. Meraj ${ }^{2}$, B. Thompson ${ }^{1,2}$ ${ }^{1}$ Newcastle University, Faculty of Medical Sciences, Newcastle upon Tyne, United Kingdom; ${ }^{2}$ Freeman Hospital, Newcastle upon Tyne Hospitals NHS Foundation Trust, Rheumatology, Newcastle upon Tyne, United Kingdom; ${ }^{3}$ Royal Victoria Infirmary, Newcastle upon Tyne Hospitals NHS Foundation Trust, Neurology, Newcastle upon Tyne, United Kingdom; ${ }^{4}$ Royal Victoria Infirmary, Newcastle upon Tyne Hospitals NHS Foundation Trust, Neurophysiology, Newcastle upon Tyne, United Kingdom

Background: Giant cell arteritis (GCA) is a systemic vasculitis affecting large to medium-sized vessels. Cranial nerve lesions are frequently documented secondary to GCA, however peripheral nerves are rarely involved.
Objectives: We present an unusual case of a GCA-associated isolated high median nerve palsy.

Methods: Case report.

Results: A 76 year old white British female presented in Spain with three weeks of frontotemporal headaches, mandibular claudication, photopsia and constitutional symptoms, followed by acute-onset right hand paresis and dysaesthesia. Acute-phase reactants were elevated. COVID-19 PCR was negative. Shortly after admission she developed acute bilateral anterior ischaemic optic neuropathy and tongue necrosis with autoamputation. Temporal artery ultrasound and biopsy confirmed GCA. PET-CT showed no evidence of a diffuse large-vessel vasculitis. She was repatriated to the UK for ongoing inpatient care. Upper limb neurophysiology two months later demonstrated an isolated right high median nerve lesion, with reduced median motor responses and absent sensory responses with denervation in extensor carpi radialis. Imaging excluded a central lesion. The timing of her symptoms suggested the lesion was secondary to GCA. Treatment was with tocilizumab, methotrexate and tapering corticosteroids, alongside PEG feeding and careful multi-disciplinary rehabilitation.

Conclusion: We suggest there are two important learning points from this complex case. Firstly, peripheral nerve lesions can occur in GCA, although they are rare. A literature search identified a modest number of case reports of brachial plexus or lower cervical nerve lesions ${ }^{1,2}$. Neurologic manifestations in GCA are attributed to vasculitis of the vasa nervorum, or extension of inflammation from arteries to contiguous nerves ${ }^{1,2}$. The restriction of clinical involvement to the median nerve alone would appear to favour the former mechanism. Functional recovery of neurological deficits is typically partial at best.

Secondly, this case highlights the difficulty faced by clinicians in recognising GCA and the importance of urgent treatment with glucocorticoids. The patien developed GCA during the ongoing COVID-19 pandemic when Spain was the second-worst affected European country (5\% seroprevalence; $95 \% \mathrm{Cl} 4.7-5.4)^{3}$ The partial overlap in symptoms between GCA and COVID-19 (e.g. headache, pyrexia, malaise, elevated acute-phase reactants) can cause diagnostic confusion and treatment delay ${ }^{4}$. GCA should be considered in the differential diagnosis of older patients with suspected COVID-19, or the non-specifically unwell elderly patient.

REFERENCES:

[1] Blaise S, et al. Horton's disease revealed by brachial C5 plexopathy [French] Rev Med Interne. 2005;26(7):578-82

[2] Merianos P, et al. Giant cell arteritis of the median nerve simulating carpal tunnel syndrome. Hand. 1983;15(3): 249-251.

[3] Pollán M, et al. Prevalence of SARS-CoV-2 in Spain (ENE-COVID): a nationwide, population-based seroepidemiological study. Lancet. 2020; 396(10250): 535-544.

[4] Monti S, Delvino P, et al. Impact of delayed diagnoses at the time of COVID19: increased rate of preventable bilateral blindness in giant cell arteritis. Ann Rheum Dis. 2020;79:1658-1659.

Table 1.

\begin{tabular}{ll}
\hline Area/movement & \multicolumn{1}{c}{ Result } \\
\hline Abductor pollicis brevis & Grade 2 weakness \\
Opponens pollicis & Grade 4 weakness \\
Pronator teres/quadratus & Grade 4 weakness \\
Flexor pollicis longus & Grade 1-2 weakness \\
Flexor digitorum profundus (digit 2 and 3) & Grade 1-2 weakness \\
Finger abduction & Preserved \\
Finger extension & Preserved \\
Other comments & \\
Dysaesthesia in distribution of median nerve & \\
\hline
\end{tabular}

Disclosure of Interests: Kundan Iqbal: None declared, James Miller Speakers bureau: Dr Miller has participated in pharmaceutical-sponsored symposiums previously but not in last five years., Consultant of: Dr Miller has participated in pharmaceutical-sponsored consultancy previously but not in last five years. Ming Lai: None declared, Mia Charnley: None declared, Muhammad Meraj: None declared, Ben Thompson Speakers bureau: Dr Thompson has participated in educational talks for Roche, Novartis, Lilly and UCB., Consultant of: Dr Thompson has participated in advisory boards for Roche, Novartis, Lilly, Gilead, Abbvie and UCB.

DOI: 10.1136/annrheumdis-2021-eular.2043 